\section{Factores de riesgo de reacción paradójica asociados a sedación endovenosa con midazolam}

\author{
MAGDALENA ESTEFÓ ${ }^{1, \mathrm{a}}$, DAGOBERTO OJEDA ${ }^{1}$, \\ PATRICIA CISTERNAS ${ }^{1}$, NICOLÁS ARRAÑO ${ }^{\mathrm{b}}$, HUGO ZANETTA $^{\mathrm{b}}$
}

\section{Paradoxical reactions associated with midazolam conscious sedation}

Background: Benzodiazepines are used for perioperative conscious sedation. However, its use may be associated with paradoxical reactions. The known risk factors for these reactions are age, alcohol and drug abuse and psychiatric disorders. Aim: To assess the incidence and impact of risk factors of paradoxical reactions to midazolam. Material and Methods: Cross sectional study of 218 patients aged $50 \pm 16$ years (51\% women) scheduled for elective surgical procedures under regional anesthesia and midazolam sedation. The paradoxical reactions were classified according to their severity in three categories. Results: The incidence of paradoxical reactions to midazolam was $8.3 \%$ (95\% confidence interval (CI) 5.0-12.7). All were mild and only $28 \%$ of the affected patients required pharmacological treatment, none of them flumazenil. A multivariable logistic regression model showed that the variables independently associated with a paradoxical reaction to midazolam were the use of psychoactive medications (Odds Ratio $(\mathrm{OR})=3.4[1.1-11], p=0.04$, and the dose of midazolam (OR 1.35 [1.03-1.78], $p=0.03$. Conclusions: The incidence of paradoxical reactions to midazolam was $8,3 \%$ and all were mild. Their risk factors are the use of psychoactive medications and the use of higher doses of midazolam.

(Rev Med Chile 2021; 149: 237-241)

Key words: Benzodiazepines; Conscious Sedation; Midazolam; Risk Factors.

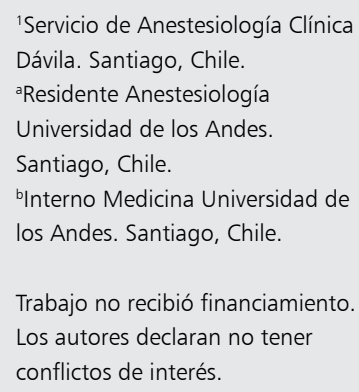

Trabajo no recibió financiamiento. Los autores declaran no tener conflictos de interés.

Recibido el 10 de junio de 2020, aceptado el 26 de enero de 2021.

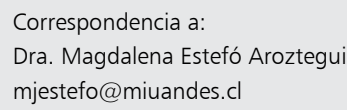

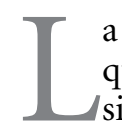

a sedación se ha descrito como un continuo que va desde la ansiolisis hasta un estado similar a la anestesia general. Se ha demostrado que la sedación aumenta la satisfacción de los pacientes y además constituye un medio para aumentar la aceptación de la anestesia regional ${ }^{1}$.

Desde su introducción en 1955, las benzodiacepinas han sido extensamente utilizadas para sedación perioperatoria y en procedimientos fuera de pabellón ${ }^{2}$, debido a que en relación a otros sedantes, exhiben un alto índice terapéutico y un gran margen de seguridad ${ }^{3}$. Las benzodiacepinas actúan por medio de estimulación de receptores de ácido $y$-aminobutírico (GABA) en el sistema activador reticular ascendente. Al estimularse estos receptores aumenta la inhibición del Sistema Nervioso Central (SNC) y se bloquean estímulos tanto corticales como límbicos', generando así sedación. De este grupo de medicamentos, destaca el midazolam por su corta latencia, brevedad de acción, potente efecto amnésico ${ }^{4}$ y que, a diferencia del diazepam, no produce dolor en la inyección endovenosa pues es hidrosoluble.

Ante la administración de benzodiacepinas, algunos pacientes en vez de experimentar sedación presentan una reacción caracterizada por desinhibición emocional, agitación psico-motora e incluso agresividad ${ }^{5}$, lo que se ha denominado 
reacción paradójica (RP). Este fenómeno es mayoritariamente idiosincrático, difícil de predecir y afortunadamente infrecuente ${ }^{1}$. Dada la variabilidad diagnóstica, la incidencia de esta reacción fluctúa entre menos del 1\% $\%^{1,6-8}$ hasta $29 \%{ }^{9}$.

Hasta el momento no hay factores causales claramente identificados que expliquen la aparición de reacciones paradójicas. Se ha elucubrado que la causa sería una variación genética en el receptor $\mathrm{GABA}_{\mathrm{A}}{ }^{6}$, lo que resultaría en una farmacodinamia alterada ${ }^{5}$.

La aparición de reacciones paradójicas frente al uso de benzodiacepinas ha sido largamente ignorada, pese a su uso difundido ${ }^{10}$. Es importante estar alerta y manejar adecuadamente estas reacciones puesto que pueden implicar riesgos y daños para el paciente en caso de ser mal manejadas. Existe escasa información en la literatura médica nacional al respecto ${ }^{11}$.

Por lo tanto, se debe mantener vigilancia activa ante la presencia de los factores de riesgo (FR) conocidos ${ }^{6}$ : género masculino, trastornos cognitivos y del ánimo, edades extremas (adultos mayores y población pediátrica), consumo de alcohol y drogas y la dosis administrada de benzodiacepinas ${ }^{5,6,7}$. Además, se ha observado un mayor riesgo de RP con el uso de benzodiacepinas de mayor potencia y menor latencia, así como las vías de administración endovenosa y tras-nasal, que posibilitan la rápida obtención de peaks plasmáticos ${ }^{2}$.

Se decidió realizar un estudio con el propósito de valorar la incidencia de las reacciones paradójicas a benzodiacepinas (midazolam), e investigar su asociación con los factores de riesgo descritos en la literatura, en pacientes quirúrgicos adultos sometidos a sedación endovenosa.

\section{Material y Método}

Se diseñó un estudio observacional de tipo corte transversal que incluyó a pacientes adultos $(>18$ años), sometidos a procedimientos quirúrgicos electivos bajo anestesia regional y sedación endovenosa con midazolam. Debido a que se reclutó a pacientes sometidos a cirugía electiva, se efectuó la evaluación previa de rigor por anestesiólogo en consulta o sala de preanestesia para garantizar que su estado de salud fuera compatible con el acto anestésico-quirúrgico y que sus enfermedades de base estuvieran compensadas. El único criterio de exclusión de los pacientes que cumplieran con estas condiciones fue rehusar a participar en el estudio. El reclutamiento de los pacientes se realizó prospectivamente entre enero y noviembre del año 2019, previa autorización del Comité Ético-Científico de la institución y firma del consentimiento informado por parte de los participantes.

Se registraron variables antropométricas y antecedentes médicos que incluían los factores de riesgo asociados a reacción paradójica (consumo de drogas, alcohol, tabaquismo, uso de psicotrópicos, antecedentes generales y psiquiátricos), el grado de clasificación de la American Society of Anesthesiologists (ASA), tipo de intervención quirúrgica, tipo de anestesia regional, dosis de midazolam y otros sedantes administrados, ocurrencia de reacción paradójica, su grado y el manejo realizado.

En el pabellón se instaló una vía venosa periférica y monitoreo anestésico estándar consistente en monitor electrocardiográfico, presión arterial no invasiva cada 5 minutos y oximetría de pulso. Como es habitual en los pacientes diabéticos quirúrgicos, se realizó una glicemia en sangre capilar previo a la administración de los agentes anestésicos, para corroborar que ésta se encontrara dentro de rangos aceptables. Se administró en forma estándar oxígeno a flujo de 3 litros por minuto por medio de una cánula nasal.

Se definió como reacción paradójica a la aparición de síntomas de inquietud, verborrea, ansiedad, agitación o a cualquier comportamiento que evidenciara un estado opuesto a la tranquilidad y adormecimiento que caracterizan a la sedación, de aparición inmediatamente posterior a la administración de midazolam, en ausencia de alteraciones hemodinámicas (hipotensión, bradicardia, arritmias) o hipoxemia.

Esta conducta anómala fue clasificada en 3 grados de severidad:

1: Locuacidad y desinhibición.

2: Agitación psicomotora moderada que no impide procedimiento quirúrgico.

3: Agitación psicomotora severa que si impide procedimiento quirúrgico.

Así mismo, se clasificó el manejo de la reacción paradójica en 4 categorías:

1. Sin requerimiento de manejo farmacológico.

2. Propofol.

3. Flumazenil. 
4. Necesidad de administrar anestesia general para controlar la agitación psico-motora del paciente y así poder continuar la cirugía.

El anestesiólogo a cargo del paciente fue quién asumió el diagnóstico, clasificación y manejo de la reacción paradójica.

\section{Análisis estadístico}

Se calculó el tamaño muestral elucubrando que la tasa de reacción paradójica aumentaría en al menos $50 \%$ en los pacientes portadores de factor(es) de riesgo, lo que redundaba en un Odds Ratio (OR) de 1,5, y resultaba en una muestra de 217 pacientes. El análisis estadístico fue realizado mediante regresión logística en el software estadístico STATA 13. Se realizó primeramente un análisis univariado considerándose como variable respuesta positiva a la presencia de una reacción paradojal y el proceso de selección de variables, (stepwise), consideró a aquellas cuyo p-valor era $<0,1$. Estas fueron incluidas en un modelo multivariado para así determinar las variables que en forma independiente y significativa se asociaran con una reacción paradójica.

\section{Resultados}

Un total de 218 pacientes fueron reclutados. La Tabla 1 resume las características demográficas de los pacientes de la muestra, la cual estuvo conformada por pacientes con un promedio de edad de $50 \pm 16$ años, principalmente ASA 2, la mayoría sometido a cirugía ortopédica y la mediana de midazolam administrado fue de $4 \mathrm{mg}$. No hubo casos de descompensaciones hemodinámicas, respiratorias ni metabólicas que pudieran haber confundido los resultados del estudio.

La incidencia de reacción paradójica a midazolam endovenoso fue de 8,3\%; IC 95\% [5,0-12,7]. Solo se registraron reacciones de grado 1 y 2 . Veintiocho porciento (28\%) de las RP requirieron manejo farmacológico, ninguno de ellos Flumazenil. Se realizó un análisis univariado que mostró que las variables con mayor asociación a reacción paradójica ( $\mathrm{p}$-value $\leq 0,1)$ fueron: Índice de masa corporal (IMC), tiempo quirúrgico, ingesta de alcohol, uso de medicamentos psiquiátricos y dosis de midazolam administrada. Estas fueron incluidas en un modelo multivariado el cual demostró que en forma independiente se asociaban a RP: uso de medicación psiquiátrica (Odds Ratio $=3,4[1,1$ 11], $\mathrm{p}=0,039)$ y dosis de midazolam administrada Odds Ratio 1,35 [1,03-1,78], $\mathrm{p}=0,032)$ (Tabla 2). En las Figuras 1 y 2 se muestran los resultados.

\section{Tabla 1. Características demográficas y basales}

\begin{tabular}{|c|c|}
\hline Variable & Resultado \\
\hline Edad (años)* & $49,4 \pm 15,8$ \\
\hline Mujeres (\%) & 51 \\
\hline IMC $\left(\mathrm{kg} \cdot \mathrm{m}^{-2}\right)^{* *}$ & $26,7(5,2)$ \\
\hline \multicolumn{2}{|l|}{ Clasificación ASA (\%) } \\
\hline 1 & 16,5 \\
\hline$\|$ & 75,2 \\
\hline III & 8,3 \\
\hline \multicolumn{2}{|l|}{ Procedimiento quirúrgico (\%) } \\
\hline Cirugía general & 4,6 \\
\hline Vascular periférico & 12,4 \\
\hline Traumatología & 39 \\
\hline Ginecología & 18 \\
\hline Oftalmología & 9 \\
\hline Urología & 6 \\
\hline Coloproctología & 11 \\
\hline Duración cirugía $(\min )^{* *}$ & $45(30)$ \\
\hline Tabaquismo (\%) & 26 \\
\hline Consumo de alcohol (\%) & 48 \\
\hline Uso drogas recreacionales (\%) & 11 \\
\hline Patología psiquiátrica (\%) & 8 \\
\hline Uso de antidepresivos y/o psicofármacos (\%) & 13 \\
\hline \multicolumn{2}{|l|}{ Posición quirúrgica (\%) } \\
\hline Supina & 55 \\
\hline Litotomía & 28 \\
\hline Prono & 9 \\
\hline Decúbito lateral & 8 \\
\hline Dosis midazolam $(\mathrm{mg})^{* *}$ & $4(2)$ \\
\hline
\end{tabular}

*Promedio \pm desviación estándar. ${ }^{* *}$ Mediana (rango intercuartílico).

Tabla 2. Análisis multivariado

\begin{tabular}{|lll|}
\hline Variable & $\begin{array}{c}\text { Odds Ratio } \\
\text { [IC 95\%] }\end{array}$ & p-valor \\
\hline $\mathrm{IMC}^{*}\left(\mathrm{~kg} \cdot \mathrm{m}^{-2}\right)$ & $1,10[0,99-1,22]$ & 0,062 \\
\hline Duración cirugía (min) & $0,98[0,96-1,00]$ & 0,100 \\
\hline Consumo de alcohol & $1,99[0,68-5,89]$ & 0,211 \\
\hline Psicofármacos & $3,42[1,06-11,02]$ & 0,039 \\
\hline Dosis Midazolam $(\mathrm{mg})$ & $1,35[1,03-1,78]$ & 0,032 \\
\hline
\end{tabular}



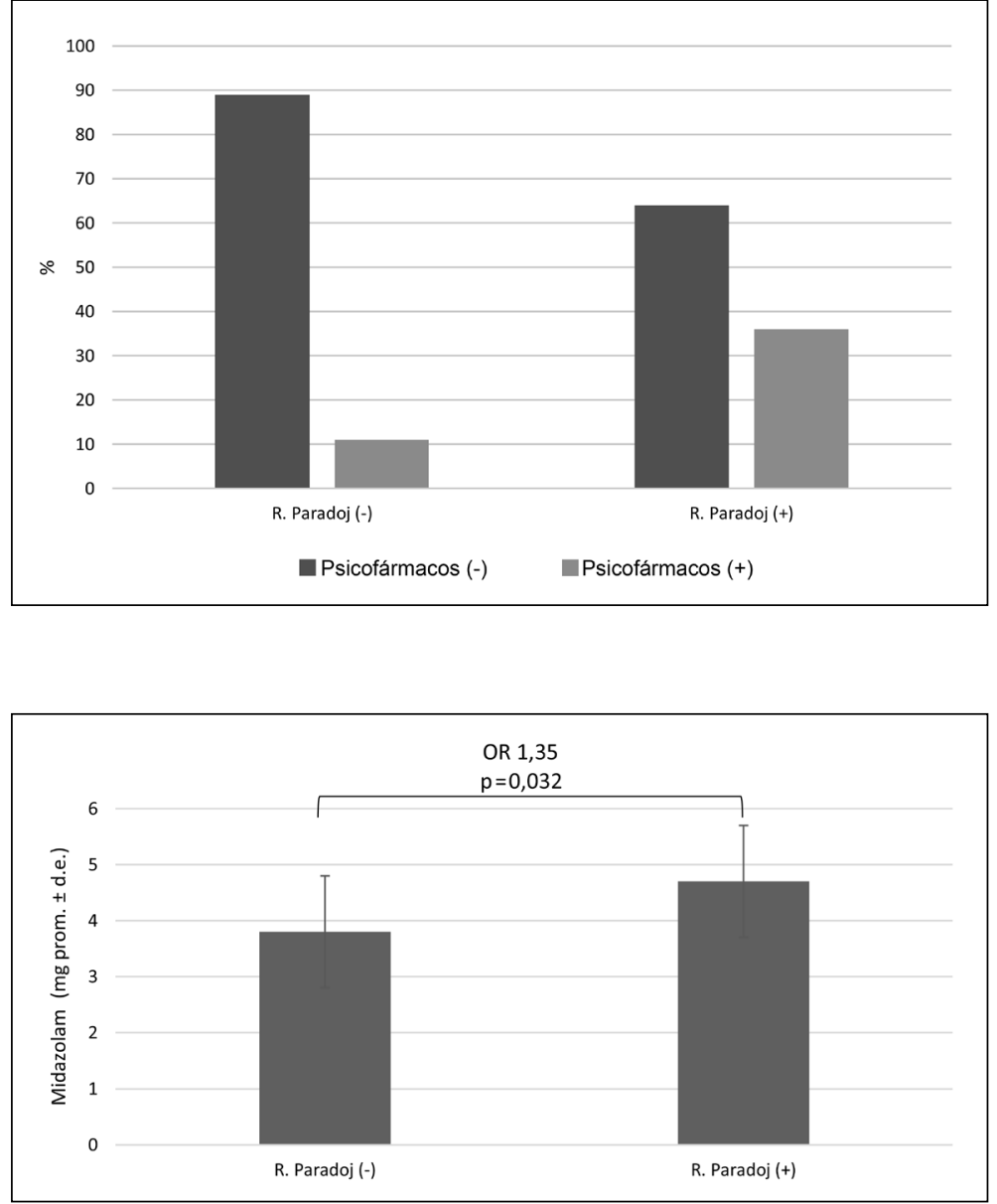

Figura 1. Incidencia reacción paradójicas según uso de psicofármacos. Odds Ratio (no ajustado) $=4,0 ; p=0,011$.

Figura 2. Dosis $(\overline{\mathbf{x}} \pm \text { d.e. })^{*}$ de midazolam y reacción paradójica.

\section{Discusión}

Los resultados de este estudio mostraron que la incidencia de RP es levemente más alta que lo descrito en la literatura ${ }^{1,5-8}$. Estudios chilenos en niños reportan una incidencia de RP de $1,5 \%{ }^{8}$. La inclusión de grados leves de RP en nuestro estudio pudiera explicar la mayor incidencia.

La asociación de la dosis de midazolam administrada y el antecedente del uso de medicamentos psiquiátricos son concordantes con lo descrito en la literatura ${ }^{6,7}$. La falta de asociación entre edad y $\mathrm{RP}$ en nuestro estudio pudiera explicarse porque la muestra estuvo conformada por pacientes entre 34 y 66 años, puesto que existe reticencia en los anestesiólogos a administrar benzodiacepinas a senescentes por el temor a los efectos adversos y a una sensibilidad exagerada a los sedantes.
Un aporte de nuestro estudio es la categorización del grado de severidad de las RP, lo que a diferencia de lo hasta ahora publicado, nos permitió reconocer que la gran mayoría de las reacciones son leves y manejables con medidas simples y no requieren terapia farmacológica. Esta falta de uniformidad en la clasificación de las reacciones paradójica podría explicar la diferencia en las tasas de incidencia reportadas en la literatura.

En conclusión, las reacciones paradójicas durante la sedación con midazolam se presentaron dentro del rango descrito en la literatura $(8,3 \%$ de los pacientes), la mayoría de ellas leves y de fácil manejo. Los pacientes en tratamiento con fármacos psicotrópicos y el uso de dosis crecientes de midazolam se asociaron a un mayor riesgo de experimentar esta respuesta. 
Agradecimientos: Sociedad Anestesiólogos San Cristóbal S.A y Servicio de Cirugía Mayor Ambulatoria (CMA) - Clínica Dávila por su contribución a la inclusión de pacientes en el estudio.

\section{Referencias}

1. Hall R, Zisook S. Paradoxical Reactions to Benzodizepines. Br. J. clin. Pharmac. 1981; 11: 99S-104S.

2. Höhener D, Blumenthal S, Borgeta A. Sedation and regional anesthesia in adult patient. Br J Anaesth 2008; 100: 8-16

3. Becker D. Pharmacodynamic Considerations for Moderate and Deep Sedation. Anesth Prog. 2012; 59 (1): 28-42.

4. Reves JG, Fragen RJ, Vinik HR, Greenblatt DJ. Midazolam: Pharmacology and uses. Anesthesiology 1985; 62 (3): 310-24.

5. Mancusso CE, Tanzi MG, Gabay M. Paradoxical reactions benzodiazepines: Literature review and treatment options. Pharmacotherapy 2004; 24 (9):1177-85.

6. Paton, C. Benzodiazepines and disinhibition: A Review. Psychiatric Bulletin 2002; 26 (12): 460-2.
7. Levene R, Su M. Paradoxical Reactions to Benzodiazepines: Who Gets Them and Why? [Internet]. American College of Emergency Physicians: Toxicology Section 2017 [cited 2019 Nov 15]. Disponible en: https://www. acep.org/how-we-serve/sections/toxicology/news/ july-2017/paradoxical-reactions-to-benzodiazepines-who-gets-them-and-why/

8. Barrionuevo L, Correa K, Consiglio M. Incidencia de reacciones adversas en sedación con midazolam endovenoso o intramuscular en niños jóvenes en situación de discapacidad. Experiencia de dos años. Rehabil. integral 2018; 13 (1): 8-13.

9. Lichtfield NB. Complications of intravenous diazepam-Adverse psychological reactions. (An assessment of 16,000 cases). Anesth Prog. 1980; 27 (6): 175-83.

10. Galleguillos T, Risco L, Garay JL, González M, Vogel M. Tendencia del uso de benzodiazepinas en una muestra de consultantes en atención primaria Rev Med Chile 2003; 131: 535-40.

11. García S, Rodríguez J, Sievers P. Efecto de la sedación en el desarrollo de delirium en pacientes adultos mayores sometidos a cirugía con anestesia espinal. Estudio clínico prospectivo. Rev Chil Anest. 2012,41:124-7. 